\title{
UNA LETRA DE CAMBIO AVALADA DE 1403
}

\author{
Rafael Conde \\ Archivo Corona de Aragón
}

El uno de diciembre de 1403 Bernat Rossell firmaba en Valencia una letra de cambio a pagar en Barcelona (1). Su formulario es el habitual, fijado ya a finales del trescientos y que perdurará durante varios siglos. He aqui el texto:

JHS.

Honrat senyor: per aquesta primera letra de cambi complirets a VIII jorns vista a.n Guillem de Baguat santanta florins d.or d.Arago, e son per altres $L X X$ florins que yo e rebuts de Bertomeu Amat; per que, senyor, al tems aga bon compliment. Feta en Valencia, primer de deembre de CCCC tres.

Lo vostre nabot Bernat Rosell, molts saluts.

Al dorso: Al molt honrat senyor en Ramon Traginer, al quarer de la Quarabasa, en Barcelona.

Primera de $L X X$ florins.

En si la letra no presenta novedad alguna. Va girada a 8 días vista, plazo habitual entre Valencia y Barcelona. Es dador del valor Bertomeu

(1) La letra de cambio y la carta que la acompaña han sido halladas en las guardas del volumen de signatura. ACA. Real Patrimonio, Bailia General, clase 8." v. 1, relativo a la recaudación de la Lleuda de Collioure. 
Amat; tomador, o librador de la letra, el firmante Bernat Rossell; pagador o librado, Ramón Traginer; beneficiaro, Guillem Bagat. El importe de la letra es de 70 florines de oro, equivalentes o al menos así se dice (2) a otros tantos recibidos en Valencia.

Bernat Rossell era desconocido en el mundo comercial y financiero valenciano y por ello precisó un aval. Procedía de Collioure, desde donde con fecha 24 de febrero de 1399 pasa a Barcelona comisionado por trebalar los negosis de la dita lleuda (de Collioure) per l.onrat Arnau Duran, titular del arriendo de la recaudación de la misma (3). Ignoramos qué estaba haciendo en Valencia en 1403, fecha de la letra de cambio. Lo vemos de nuevo en Barcelona en 1406 y 1411 (4).

Del resto de los implicados en la operación tenemos datos dispersos. Bertomeu Amat era comerciante, y lo vemos en 1418 como librador de una letra de cambio a favor de Pedro Palomar, mercader genovés residente en Barcelona, letra que fue protestada (5).

Guillem Bagat se halla algo más documentado. Era mercader, ciudadano de Barcelona y procedía de Montpellier. Carrère lo encuentra a finales del trescientos asegurando aludas y cordobanes por valor de 132 libras barcelonesas para Génova y en 1407 en una operación financiera (6).

Ramón Traginer me es conocido únicamente a través de otras letras de cambio de los años 1404 y 1406 pertenecientes al mismo conjunto que la que me ocupa. Aparece como librador de dos letras por valor de 50 florines y 22 libras, respectivamente, enviadas desde Perpignan contra nuestro mismo Bernat Rossell.

(2) En nuestra letra de cambio la cantidad a pagar equivale, teóricamente, a la recibida. Como algún beneficio debe obtener el dador del valor, es licito pensar que hubo un descuento previo.

(3) Según se hace constar en la apertura del volumen en cuyas guardas ha aparecido nuestra I.d.c. Por sus gestiones se le asigna un salario de $\mathbf{8 0}$ libras anuales. Rossell se convierte en un agente de Duran durante varios años y su actividad al margen de los afers de la leuda a que se hace referencia, queda recogida en alguno de los volúmenes de la serie que recoge asientos relativos a operaciones comerciales y financieras.

(4) Como nos documentan otras letras de cambio halladas en las mismas guardas.

(5) C. CARRERE, Barcelone, centre économique à l'epoque des difficultés. 1380-1462, Paris-La Haye, 1967. Trad. catalana: Barcelona 1380-1462. Un centre econdmic en época de crisi, Barcelona, 1977. Cito por la edición catalana. Las noticias sobre Bertomeu Amat en vol. II, pág. 158.

(6) C. CARRERE, Barcelona, cit. vol. II, págs. 47 y 283. Y como garantía en un contrato de aprendizaje de Ramón Vidal con Tomás Sala, el primero de Montpellier y el segundo de Barcelona, en documento no fechado (íbid., vol. I, pág. 143). 
La letra presenta la particularidad de ir avalada y va acompañada de una "lettera di contenta" del avalista al librador que relata la historia de la operación.

La bibliografía disponible permite trazar los rasgos de la figura del avalista (7) quinta que se une a los cuatro vértices que configuran el complejo mecanismo de la letra de cambio medieval.

La forma normal de la expresión del aval consiste en la mención del mismo, manuscrita del avalista, al pie de la letra de cambio. En virtud de esta suscripción, el avalista garantiza al dador del valor la recuperación del mismo. Existen otros tipos de garantías. Cassandro documenta la entrega de un pignus por parte del librador para garantizar los cambios. Concretamente se hace eco de un pleito de principios del siglo $\mathrm{XVI}$ sustanciado o iniciado ante el Sacro Consiglio napolitano, derivado de una letra de cambio garantizada con un bien mueble. Se planteaba si el dador del valor debía conformarse con el pignus o beneficiarse del recambio de la letra impagada. Convocado el Sacro Consiglio, se interroga a los comerciantes para conocer su parecer. Un tal Taddeo de Alborea declara que «quando se danno ad cambio et non nce pigno corre lo periculo de perder li denari, ma quando ncè pigno non si debe guadagnar lo recambio, quando li denari non se pagano». En cambio la

(7) R. DE ROOVER se ocupa del aval tan sólo en L'Evolution de la lettre de change. XIVe-XVIIIe siècles (Paris, 1935) aunque de forma tangencial y en un sentido totalmente distinto. Hablando de los problemas del origen del endoso, y según los "doctores" del XVII, la palabra «aval» es utilizada en letras en que el librador "reconnait avoir reçu la valeur de Paul pour Jean pour Pierre pour le compte de Nicolas (...) II s(agit donc toujours d'operations de change en filiere, conclues probablement dans un but spéculatif" (págs. 84-85). Está claro que el término tiene en este contexto un sentido totalmente diverso al que nos ocupa y que es el corriente. En el resto de sus exposiciones teóricas sobre la letra de cambio olvida la figura. Al igual que Federigo MELIS en su Documenti per la storia economica dei secoli XIII-XVI (Firenze, 1972). Del aval hablan Giovanni CASSANDRO en Vicende storiche della lettera di cambio (Bolletino dell'Archivio Storico, Banco di Napoli, 9-12, 1955-1956, págs. 1-91): C. TRASSELLI en Note per la storia dei banchi in Sicilia nel XV secolo, Parte II: I banchieri e i loro affari (Palermo, 1968) en las páginas referentes a la descripción de la letra de cambio del ámbito siciliano; y H. LAPEYRE en Alphonse $V$ et ses banquiers (Moyen Age, LXVII, 1961, págs. 93-136) y en Contribution a l'histoire de la lettre de change en Espagne du XIVe au XVIIle siècle (Anuario de Historia económica y Social, I, 1968, págs. 107-125). La bibliografía disponible sobre la letra de cambio catalana o en el ámbito catalán está muy dispersa y es puntual. Se encuentra recogida en mi trabajo Seis letras de cambio cuatrocentistas giradas contra Barcelona (Estudios Históricos y Documentos de los Archivos de Protocolos, V, Homenaje en honor de Josep Maria Madurell y Marimon, Barcelona, 1977) Vide et. la reedición de los trabajos de Andre Sayous sobre las técnicas comerciales barcelonesas en la Edad Media (E/s métodes comercials a la Barcelona medieval, Barcelona, 1975) y la bibliografía allí recogida por los autores del estudio introductorio Arcadi Garcia i Sanz y Gaspar Feliu i Montfort. 
mayoría se pronunció en sentido opuesto, pues el pignus es algo extraño al cambio, ya que lo que gobierna el sistema es la "contenta": "acteso maxime che dice per altri tanti avuti", tal como afirma Bernardino Scaglia, otro comerciante convocado a declarar (8).

La práctica de garantizar una letra de cambio con un pignus es anterior. Alfonso el Magnánimo garantizaba cambios de Italia contra su tesorería por este sistema: en una carta dirigida a su tesorero, de $27 \mathrm{de}$ noviembre de 1448 , le ruega pague los cambios que le envía, pues tiene en "Penyora ... joyas, oro, argent e quanto tengo", que tendrán que venderse si no se realiza el pago (9).

El mismo Alfonso utilizaba otra forma de garantizar sus cambios, y era el compromiso formal en documento aparte, a favor del dador del valor (10).

Observemos que en los tres casos (suscripción de la letra, pignus y compromiso en documento aparte) la garantía se ofrece en la plaza de origen a favor del dador del valor, no a favor del beneficiario de la letra como en la práctica actual.

Recordemos brevemente que en una letra de cambio actúan cuatro personajes: en la localidad $L$ el dador del valor presta una cantidad a un tomador que le hace entrega de un título de crédito, la letra de cambio, por el que éste, librador, ordena a su agente en la localidad $L^{\prime}$ que pague a un beneficiario, agente del dador del valor, esta misma canti-

(8) G. CASSANDRO, Vicende storiche, cit., págs. 40-42.

(9) Tesorero: vuestras letras e recebidas: cuanto a la nao, no quiero que torne a Barcelona, sino que quando sera cargada de sal se venga con la ayuda de Dios a Naples. Soy maravillado como no me haveys fecho mencion de los cambios, si son pagados todos o parte, ni quales, porque yo tengo en poder de los mercaderes por su seguretat joyas, oro, argent e quanto tengo, e si tornan los cambios a mi, seria grandissimo danyo e se haverian vender las penyoras, e asi con cada correu me scrivit de lo que sobre esto fareys, alla vos he enviado los partidos de que podreys pagar e agora vos los torno a scrivir por dos o tres partes por que al menos hayays los unos. El desempachamiento de Rertran Crexe/ls fazet que lugo haya desempatchamiento si no lo avia. Scrita de mi mano en Napols a XXVII de novembre. Rex Alfonsus. Fazet con suma diligencia que Crexells sia presto pagado de la assignacion de los pageses de remença e do no uviese manera dalde de otra parte considerando quanto danyo me seria el tornar de los dichos cambios. A mi tesorero mossen Perot Mercader. (ACA. Cancilleria, reg. 2940, fol. 59)

(10) Vide p.e. las prestadas a Berto Agosti: Yo el rey de Aragon e de las Dos Sicilias, prometo e juro a Dios e a los santos cuatro evangelios corporalmente tocados a ti, Berto Agosti, que si caso es que un cambio de dos mil ducados que Jaume Gil, de mi thesoreria, a preso por mi de ti, Berto Agosti para Barcelona a cuatro meses fecha, a razon de diez y seys sueldos e seys dineros por ducado, el qual cambio fue fecho a XIII de mayo, que torne e no sera pagado, tornado que sea aqui con todos los intereses que vernan, yo los pagare; e por seguredad tuya te fago la presente escrita de mi mano e sillada de mi anillo en Tybuli a XIII de mayo anno mil CCC XXXX VII. Rex Alfonsus. (ACA. Cancillería, reg. 2940, fol. $29 \mathrm{v}^{\circ}$ ) 
dad en moneda propia de la localidad. El dador del valor envía uno o más ejemplares de la orden de pago al beneficiario, el cual presenta la letra al cobro al librado en el término o plazo expresado en el documento, este lo acepta y lo paga. En este momento el dador del valor, el que ha prestado el dinero en la localidad $\angle$ tiene su capital expatriado y debe repatriarlo. En palabras de Roover, una operación de cambio no estaba completa hasta que el dador del valor "fassait ses retours" (11). La repatriación del capital prestado puede hacerse por medios extracambiarios, como en mercancías, en monedas, en metal precioso (12), y por medios cambiarios: una letra de cambio desde la localidad $L$ a $L^{\prime}$ a su favor.

Quien arriesga, pues, el dinero, es el dador del valor, y por lo tanto, es lógico que éste quiera seguridades de su recuperación. En la plaza de destino tiene dos mecanismos jurídicos para garantizar la recuperación del dinero por parte de su agente: que el beneficiario, en caso de impago, inicie una acción legal contra el librado (13) o que acuda al protesto. En este segundo caso el beneficiario le remitirá una letra de cambio, el recambio, contra el librador que se convertirá en librado, y a favor del dador del valor. En caso de no confiar en el tomador del dinero o librador de la letra, exigir a éste un aval a su favor y en su plaza.

En opinión de Lapeyre, el aval sirvió para pactar la ricorsa de la letra de cambio (14) es decir, el pago de la misma, no en la plaza de destino, sino en la de origen.

La fórmula de este aval, que estudia Lapeyre en una letra de cambio de 1702, impreso su formulario y el del aval, se concreta en "Digo yo N.N. que me obligo en el presente cambio y recambio como principal tomador, pagador y deudor..." A continuación se especifica que el dador del valor designará la persona que pagará la letra al término del plazo en la feria de Medina del Campo (plaza de destino) y que el avalista pagará la letra de recambio en Valencia (plaza de origen). Se precisa que el dinero dado a cambio es entregado a la persona que firma la primera letra, es decir, al librador, disposiciones que son carac-

(11) R, DE ROOVER, L'evolution, cit., pág. 54.

(12) Ibid., pág. 54 y The rise and decline of the Medici Bank, Cambridge Mss. 1963. Trad. italiana II Banco Medici dalle origini al declino (1397-1494) Firenze 1970, pág. 160. Cito por la traducción italiana.

(13) Al menos en la práctica mallorquina según A. GARCIA SANZ, Els origens del dret canviari català en Miscel.lania Historica Catalana. Homenatge al Pare Finestres, Historiador de Poblet ( $\dagger$ 1769) Abadía de Poblet, 1970, pág. 228.

(14) H. LAPEYRE, Contribution, cit., págs. 118-122. 
terísticas la de ricorsa (15). El mismo Lapeyre recoge otro ejemplo anterior, de 1524 , en que el avalista se obliga "al cambio y recambio... como principal tomador o pagador" en un caso también de ricorsa (16). Y aún podriamos hipotetizar en torno a un caso de 1461 recogido por Nuria Coll (17). Se trata de una letra de Barcelona a Brujas con la fórmula (pagau) a vos matex que aparece en casos de ricorsa, y en que tal vez el subconsciente juega una mala jugada al avalista quien suscribe: Jo, Francesch Oller, so tengut en lo present cambi com a principal (tachado pagador) prenedor. El paralelismo con los avales estudiados por Lapeyre es evidente.

En virtud de la exigencia hecha a Rosell de presentar un avalista, Tomaso Biliotti escribe de su puño y letra y al pie del documento:

\section{Jo, Thomas Biliotti, son thengut del dicto chambio}

Biliotti pertenece a la casa florentina Piacitti-Biliotti, una de las muchas que se instalan en Valencia a fines del siglo XIV, bajo el reinado de Martín I, en el momento de auge y crecimiento de la presencia florentina en la Corona de Aragón (18). Mario del Treppo cita un Tomaso di Gualterio Biliotti como arrendador de la ceca valenciana bajo el mencionado rey Martín (19). No he podido hallar vinculación alguna entre Rossell y Biliotti que justifique la disposición del último a avalar la letra. Posiblemente han mediado garantías notariales por parte de Rossell para que el italiano consienta en poner su firma en el documento (20).

La fórmula de prestación del aval es la corriente en la época, tanto en el ámbito catalán como en el italiano, por más que, normalmente se precisa "com a principal prenedor" 0 “come principale prenditore" (21).

(15) Ibíd., pág. 120 y Apéndice doc. VI, pág. 125.

(16) Ibíd., pág. 120 y Apéndice, doc. V, pág. 124.

(17) N. COLL JULIA, Una Letra de cambio y de su recambio originales de 1461; transferencia de la segunda, en Estudios Históricos y Documentos de los Archivos de Protocolos, IV, Miscelánea en honor de Raimundo Noguera de Guzmán, Barcelona, 1974, págs. 447. 454.

(18) M. T. FERRER I MALLOL. E/s italians a les terres catalanes (segles XII al XV), en "Anuario de estudios medievales" 10 (1980) Actas del I Congreso Internacional de Historia Mediterránea (Palma de Mallorca, 1973), págs. 393-469.

(19) Junto con Matteo di Buonaccorso, por 2.000 florines, en fecha no precisada, en $M$. DEL TREPPO, I Mercanti Catalani e l'espansione della Corona d'Aragona, Napoli, 1972, pág. 271. Un Tomaso Biliotti es mencionado por Usher como firmante de órdenes de pago contra la banca Datini a favor de Filipo di Gualterio Biliotti (The Early History of Deposit Banking in Mediterranean Europe, Cambridge Mss. pág. 93).

(20) A estas garantías hace referencia N. COLL en Una letra de cambio, cit., pág. 449.

(21) Cf. N. COLL JULIA, Una letra de cambio, cit., págs. 449-450: H. PIRENNE, Alphonse V et ses banquiers, cit., pág. 98 y Contribution, cit., pág. 118; o similar: Yo, Arnau Fonolleda, 
Sayous en sus clásicos, aunque ya superados, trabajos sobre los métodos comerciales en la Barcelona Medieval (22) no comprendió el significado del término prenedor y creyó, erróneamente, solucionar su significado al interpretar mal una equivalencia dada por Moliné en su edición del «Llibre de Consolat de Mar». En su glosario, Moliné i Brases traduce prenedor como prestatari en un contexto no cambiario (23). Sayous interpretó mal el prestatari, pasivo y coherente con el contexto en que aparece la palabra prenedor, por prestador, activo. Para, naturalmente, dejar sin respuesta el qué significa la presencia de un «principal prestador" en una letra de cambio. Y problablemente, Sayous no podía ni siquiera comprender por qué había un prestador. Al no hallar descuento en las letras de cambio negó función financiera al título. Por ello, tras recoger la identificación, acertada, que los organizadores de la exposición de documentos mercantiles realizada en Barcelona en 1932 hicieron del término como aval (24), afirma que, por lo menos en una letra de cambio de 1462 , el término prenedor no puede significar aval pues la letra procede de Esmirna, y allí se firma el aval, y debe pagarse en Barcelona. Lo cual, bajo la perspectiva apuntada en estas líneas es lo lógico.

Tampoco Usher acabó de identificar al prenedor. En el trabajo que publicó en 1931 (25) afirma que aparecen en las letras anotaciones que indican que una cuarta persona (en realidad debería decir una quinta)

secretari de/ senyor rey, me scrich en lo present cambi com a principal prenedor en letra de cambio de Alfonso $\mathrm{V}$ contra Berenguer d'Oms, gobernador de Mallorca, reproducida en C. CARRERE, Barcelona, cit., lám. fuera de texto. Los italianos reflejan la misma fórmula: Noi Zanobi Peruzzi et compagni siamo contenti et essere tenuti a lo supradicto cambio come principali prenditori (G. CASSANDRO, Vicende storiche, cit., pág. 42), en letra de cambio de Roma de 1447; Jo Zacheo di ser andrea mi obrigo al sopra dicto cambio chome principale prenditore et si chontento che li dicti denari sieno dati al sopradecto gioanni matteo (C. TRASSELLI, Note cit., pág. 33) en letra de cambio de 1470. Los avales castellanos conocidos, del siglo XVI, se mueven en la misma órbita (cf. H. LAPEYRE, Contribution, cit., doc. Apéndice $V, V I)$.

(22) A. SAYOUS, Els mètodes comercials, cit., pág. 126.

(23) E. MOLINE I BRASES, Les costums maritimes de Barcelona universalment conegudes per "Llibre de Consolat de Mar" (Barcelona, 1914), pág. 287 refiriendo el prenedor al capítulo CCCXXVIII que habla de los préstamos que el "almirall» hace a los marineros, que se convierten asi en prenedors dels diners.

(24) Catàleg de l'Exposició retrospectiva de documents mercanti/s organitzada per l'Associació de Contables de Catalunya, dec. 1932 (Barcelona, 1932), doc. 282 del catálogo: aval de Martín Gómez de Perapertusa, de Zaragoza, en una I.d.c. de 1462.

(25) A. P. USHER, Deposit banking in Barcelona 1300-1700, en Journal of Economic and Bussines History, IV, 1931 (Trad. catalana con título en castellano, La banca de depósito en Barcelona, 1300-1700, en Cuadernos de Historia Económica de Cataluña, Segundo Trimestre del curso 1969-1970), págs. 157-181. 
acepta la letra. El verbo tenir y la palabra prenedor, tomadas literalmente, continúa, podrían significar la cesión por parte del tenedor, es decir, un endoso. Pero él mismo reconoce que del contexto de algunas letras más bien parece sobreentenderse que una cuarta persona ha aceptado las responsabilidades dimanantes de la letra. Cita letras de diversas fechas del XV, una de las cuales avalada por un tal Arnau Fonolleda con la fórmula habitual, en cuyo comentario afirma que no admite otra interpretación que la de librado. Usher roza continuamente la identificación pero no pronuncia la palabra aval. Ni siquiera en su monumental obra sobre la banca de depósito en la Europa mediterránea, donde, en su glosario colocado en apéndice, traduce el prenedor como "taker (ie. acceptor or buyer) of a bill of exchange" (26).

El avalista sustituye, pues, en última instancia al librador o tomador en la devolución del dinero al dador en caso de impago. Endemann pretende, basándose en el hecho de que se avalan las segundas y terceras de cambio, que el avalista renueva la obligación del librador. Argumenta Cassandro contra él que es normal avalar las segundas y terceras y que, por lo tanto, el aval no significa una renovación de la obligación. Cita dos textos: uno del "Tractatus de cambiis" de Raphael de Turri y otro de las Ordenanzas boloñesas de 1567 (27) y aduce el testimonio de Bensa referente a las letras del archivo Datini. Concluye Cassandro que el avalista es responsable a la par que el emitente o librador (28). De hecho la norma de avalar segundas y terceras no es universal. Biliotti avala una primera y hay otros casos (29). El hecho de que hallemos segundas y terceras avaladas no significa que no se haya avalado la primera. El no avalar todos los ejemplares de la letra resulta inexplicable pues lo duplicado y triplicado son sustitutos de la primera y tienen su misma fuerza ejecutiva.

(26) A. P. USHER, The Early History of Deposit Banking, cit., págs. 683-584.

(27) Del siglo XVI: ... occurrit ille modus quo contrahentes cambium, ut magis sit cautum creditori, conveniunt ut alius, preter debitorem, subscribat secundas litteras cambii (Raphael de Turri, Tractatus de cambiis, Disp. I, Quaest. XVII) y ... alle volte che alcuno dà denari a cambio, lo fa con haver secortà, la quale sottoscrive la seconda o la terza di cambio come si costuma in tutte le piazze (Ordenanzas boloñesas aprobadas por el Papa el 25 de noviembre de 1569) recogidas por Raphael de Turri, op. cit. Ambos textos en G. CASSANDRO, Vicende Storische, cit., pág. 42.

(28) G. CASSANDRO, Vicende Storische, cit., págs. 42-43.

(29) Es también una primera la que avala Bartolomeo Bonconti en 1470 (C. TRASELLI, Note cit., pág. 30), la que avala Arnau Fonolleda en 1444 (vide supra nota 21), las que avalan Jacme Carreres y Berenguer d.Espasen en 1448 (R. CONDE, Un episodio cambiario entre Palermo y Barcelona (1448) (en prensa), etc. 
Aceptada la explicación del término prenedor, como sustituto del emitente o librador de la letra, queda por explicar la "principalidad" del prenedor. ¿Por qué "principal" cuando realmente el principal tomador es quien ha recibido en préstamo el importe de la letra de cambio y es, por lo tanto «principal» responsable de su devolución?

La fórmula del aval de Biliotti está en la línea de las ordinarias, excepto que simplificada, pues se limita a considerarse como tengut, obligado. No es ésta la única nomenclatura usada. Se utiliza también el término fermança o garantía (30) y como tal se identifica el mismo Biliotti en la lettera de contenta que acompaña a la letra.

La letra fue presentada al cobro, pero el librado estaba ausente de Barcelona. Por eso falta en el documento la cláusula de aceptación, reglamentaria desde la ordenanza barcelonesa de 1394 que establecía que debía hacerse constar por escrito en la misma letra de cambio en el plazo de 24 horas a partir del momento de presentación (31). La historia de la operación que nos ocupa puede seguirse, a partir de este momento, a través de la nota manuscrita de Bagat en el documento y a través de la lettera di contenta firmada por Biliotti.

En la letra, Guillem Bagat se declara satisfecho de los 70 florines: Jo, Guillem de Bagat son stat content del dit cambi a XXIIII de dezembre 1403, e los dits 70 florins he ahuts d'Andrea di Pazzi per ço car en Ramon Traginer s. en era anat e no ych avia negun qui.m volgues respondre per ell.

La parte final del texto hace referencia sin duda a la costumbre mercantil denominada en italiano "onorare la lettera» (32). Y puesto que nadie quiso "honrar" la letra, quedaba abierto al beneficiario el camino del protesto y del recambio. En el recambio Rossell se convertiría en librado y Amat en beneficiario. En caso de impago de este recambio, caso plausible dado el carácter de forastero de Rossell $-\mathrm{y}$ por esto se le pidió aval- Biliotti pagaría a Amat y luego se revolveria contra Rossell.

La letra fue pagada en Barcelona, en ausencia del librado y en ausencia lógica del avalista, por el italiano Andrea de Pazzi amich meu tal como lo llama en la "contenta» Biliotti, cuyo texto, en un catalán fuertemente italianizado, reza:

(30) H. LAPEYRE, Alphonse $V$ et ses banquiers, cit., pág. 99. $Y$ en una interesantísima letra de cambio de 1399, de Venecia a Barcelona, letra que es de recambio por una l.d.c. no pagada por el librado y de cuyo importe se hizo cargo el avalista leemos: $e$ yo lin fet compliment per farmansa que li.n fuy, conservada en el Instituto Municipal de Historia de Barcelona y reproducida en Catàleg de l'Exposició retrospectiva, cit., doc. 269.

(31) A. GARCIA SANZ, E/s Origens del dret cambiari catala, cit., págs. 227-228.

(32) G. CASSANDRO, Vicende Storiche, cit., pág. 45. 
IHS.

Jo Tomas Biliotti Biliotti, merchader floranti, rebi. per esta de III de sattembre 1404 da vos, senyor en Brnat Rossel de Colliura florins vintinou $X X V I I I I^{\circ}$ sous $V$ del quals vos m.ets tenghut per una letra di chambi che jo paghi per vos come apresso dire. Jo vi fu fermanza a una letra de chanbi de florins $L X X$ che vos prendest qui a chanbi a di 1 de deembre 1403 dal senyor en Berthomeu Amat e adrizasts la vostra letra a Barcelona al senyor en Ramon Tragginer $[c]$ he co $[m] p / i s$ al senyor en Ghislem de Baghat e (...) detto [s] enyor en Ramon Traginer non fu trobat en Bar[celon]a e per zo che jo ere fermanza per vos, en An[d]rea de Pazzi, amich meu, conpli per vos lo dit chanbi e presi lo richanbi de Franciescho Manelli e chompany ch $[e$ f]uron ab cost e dams e mession florins LXXII sous II, lo qual rec[ha]nbi v.envio qui per paghar e Girolamo di Leonardo [m] erchader vinizian, e jo conpli qui per vos al dit Gi rolamo los dits LXX/I florins e II sous, e depuis lo dit senyor en Ramon Traginer conpli per lo dit cha[n]bi al senyor en Johan Fabre de Pirpignan lliures $[X] X I I I$ sous $X$ de barzalonesos e pero, senyor, me restat a donar florins $X X V I I I I^{\circ}$ sous $V$ de/ quals jo son contento e paghato da vos, senyor en Brnat supradicto como damut es dit. E per [s]o vos fo fine del dit chanbi e o fac per esta contenta de ma mia propio mano lo damunt d[it] jorn. Thomas Biliotti in Valensia.

Al dorso: Contenta fetta per me Thomas Biliot al senyor en Bernat Rossel.

De otra mano: al mot (?)

Una vez Pazzi ha pagado en nombre de Biliotti, se revuelve contra él por medio de otra letra de cambio, calificada en el texto, un tanto impropiamente, de recambio. Su importe asciende a 72 florines y 2 sueldos, es decir, los 70 florines originarios y 2 florines y 2 sueldos en concepto de cost e dams e messions, gastos que, no habiendo mediado protesto, cabe atribuir únicamente a lo percibido por el corredor de cambios que intervino en la operación (33) y a los gastos previstos de correo. Dador del valor fue Francesco Manelli, florentino y harto conocido en Barcelona (34) y con quien Pazzi mantiene relaciones comercia-

(33) G. CASSANDRO, Vicente Storische, cit., págs. 44-45; R. DE ROOVER, // Banco Medici, cit., pág. 163.

(34) Sobre los Manelli y sus actividades en Barcelona, cf. M. DEL TREPPO, / Mercanti, cit., var. loc. Sobre Andrea de Pazzi, ibid., var. loc. 
les y financieras; librador de la letra Andrea de Pazzi; librado, naturalmente Biliotti y beneficiario, el veneciano Girolamo di Leonardo, agente o corresponsal de Pazzi. No se ha conservado la letra, pero fue aceptada y pagada según declaración de nuestro avalista.

Se abre ahora una tercera etapa: la recuperación de los 72 florines y 2 sueldos de Bernat Rossell por parte de Tomaso Biliotti. El documento, la contenta de Biliotti, nos explica el proceso.

La recuperación se hizo fragmentada. En las primeras líneas de la carta, Biliotti se considera pagado por Bernat Rossell de 29 florines y 5 ss. barceloneses sin indicar cómo. Las últimas líneas de la misma carta nos indican que Ramón Traginer pagó a Joan Fabre de Perpignan, 23 libras y 10 sueldos barceloneses. Aunque el texto no autoriza una declaración definitiva, tanto el vocabulario como otras circunstancias (la "differentia loci" entre Valencia y Perpignan) permiten sospechar que el pago se realizó por letra de cambio.

En conjunto la operación fue breve. Duró desde el 1 de diciembre de 1403 al 3 de septiembre de 1404, tan sólo 10 meses. La califico de breve comparándola con un caso napolitano, de mitad del $X V$, que estudia Cassandro, en el cual el avalista, Zenobi Peruzzi, tardó 21 años en recuperar del librador, Carlo Caracciolo, la cantidad que se había visto obligado a pagar como avalista. $Y$ aún, como destaca Cassandro, porque Caracciolo se hace oblato del hospital de la Anunziata de Nápoles y renuncia a una enésima reclamación contra la sentencia del Sacro Consiglio de Nápoles que le condenaba. La letra fue de 1447 y la liquidación de 1468 (35).

(35) G. CASSANDRO, Vicende Storische, cit., págs. 42-43. 